\title{
DELAYED-ONSET RHABdomyolysis RELATED TO OLANZAPINE: A CASE REPORT
}

Singapore Med J 2016; 57(5): 279 doi: 10.11622/smedj.2016094

Dear Sir,

Olanzapine is a widely used, atypical antipsychotic. Few reports on the association between regular dosage of olanzapine and rhabdomyolysis have been published to date. The present case report draws attention to delayed-onset rhabdomyolysis, a rare adverse effect of olanzapine therapy, and discusses its possible mechanisms.

The patient, a 62-year-old man, had a history of chronic schizophrenia, with his first episode of auditory hallucination and persecutory delusion occurring at 25 years of age. Between the ages of 30 and 60 years, the patient was repeatedly hospitalised due to psychotic exacerbations. About two years prior to the current presentation, he was receiving rehabilitation in a halfway house and treated with olanzapine $10 \mathrm{mg} /$ day, valproate $500 \mathrm{mg} /$ day, estazolam $2 \mathrm{mg} /$ day and benzhexol hydrochloride $2 \mathrm{mg} /$ day.

The patient was sent to our emergency department when he could not get up from the floor due to generalised weakness and paralysis. On presentation, he was conscious, and had decreased muscle power but no extrapyramidal symptoms. His creatine kinase (CK) level was high (13,046 U/L), but troponin, heart type creatine kinase isoenzyme, blood urea nitrogen, creatinine, complete blood count, electrolytes, C-reactive protein and glucose levels were all normal. Rhabdomyolysis was diagnosed.

On suspicion of its contribution to rhabdomyolysis, olanzapine was stopped. Following hydration, the patient's CK level fell to $11,407 \mathrm{U} / \mathrm{L}$ three days later and he was discharged back to the rehabilitation facility on the fifth day. At the one-month outpatient follow-up, his CK level was within normal limits.

The patient had no evidence of seizure on clinical presentation. He also did not have fever or muscle rigidity, making neuroleptic malignant syndrome unlikely. The causal relationship suggesting that olanzapine was the probable cause of rhabdomyolysis was supported by a score of 5 in the Naranjo Adverse Drug Reaction Probability Scale. ${ }^{(1)}$ There have been previous case reports of olanzapineinduced elevation of serum CK level occurring within seven weeks of administration. ${ }^{(2,3)}$ In our case, olanzapine 10 mg/day was administered for nearly two years without dose titration, which could have led to delayed-onset, olanzapine-related rhabdomyolysis. However, the combined effects of polypharmacy might also be a contributory factor, since olanzapine was used concomitantly with other medications like valproate, estazolam and benzhexol hydrochloride. The patient might have been vulnerable to some incipient muscle changes related to the use of olanzapine or the combination of medications over the two years, prior to the occurrence of drug-induced rhabdomyolysis.

Although unclear, the mechanism of olanzapine-related rhabdomyolysis might be drug-related myopathy. Olanzapine is an atypical antipsychotic with a high affinity for histamine-H1, 5-hydroxytryptamine 2A (5-HT2A) and dopamine D2. H1-receptor antihistamines, acting on the sarcolemma, may facilitate sodium flux into the cells, thereby depleting intracellular adenosine triphosphate (ATP) through the activation of energy-dependent $\mathrm{Na}+\mathrm{K}+\mathrm{ATPase}$. Furthermore, an increased intracellular sodium concentration can elevate calcium and activate intracellular proteolytic enzymes, causing progressive rhabdomyolysis-related injury to muscle cells. ${ }^{(4)}$ Also, the antagonist activity at the 5 -HT2 A receptors can block the uptake of glucose by skeletal muscles and increase its permeability to CK. ${ }^{(5)}$

In conclusion, even in patients receiving long-term olanzapine treatment, the possibility of delayed-onset rhabdomyolysis should be considered. If clinical manifestations such as muscle weakness, fever or alternations in consciousness occur, monitoring of serum $\mathrm{CK}$ is required.

Yours sincerely,

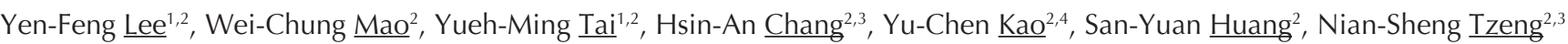

${ }^{1}$ Department of Psychiatry, Tri-Service General Hospital - Beitou Branch, ${ }^{2}$ Department of Psychiatry, Tri-Service General Hospital, ${ }^{3}$ Student Counseling Center, ${ }^{4}$ Department of Psychiatry, Tri-Service General Hospital - Songshan Branch, National Defense Medical Center, Taipei, Taiwan ROC. pierrens@mail.ndmctsgh.edu.tw

\section{References}

1. Naranjo CA, Busto U, Sellers EM, et al. A method for estimating the probability of adverse drug reactions. Clin Pharmacol Ther 1981; 30:239-45.

2. Perlov E, Tebartz van Elst L, Czygan M, Bubl E, Ebert D. Serum creatine kinase elevation as a possible complication of therapy with olanzapine. Naunyn Schmiedebergs Arch Pharmacol 2005; 372:168-9.

3. Punukollu B, Rutherford H. Serum creatine kinase elevation associated with olanzapine treatment. BMJ Case Rep 2008; $2008: b c r 0620080040$.

4. Khosla U, Ruel KS, Hunt DP. Antihistamine-induced rhabdomyolysis. South Med J 2003; 96:1023-6.

5. Meltzer HY. Massive serum creatine kinase increases with atypical antipsychotic drugs: what is the mechanism and the message? Psychopharmacology (Berl) 2000; 150:349-50. 